\title{
Encoding and performance in sentence verification under varying memory load
}

\author{
THOMAS H. CARR \\ University of Oregon, Eugene, Oregon 97403 \\ and \\ VERNE R. BACHARACH \\ Acadia University, Wolfuille, Nova Scotia
}

\begin{abstract}
Subjects verified verbal descriptions of the forms "This (is, is not) a (color) (shape)" and "This (is, is not) a (shape) that's (color)" against two-dimensional geometric figures. The figure was seen immediately after the description was heard or after a filled delay. Latency and error data indicated that differences in surface-structure organization affected encoding but not comparison. Some subjects appeared to transform negative conjunctions into positive disjunctions before comparison. More subjects showed this transformation under delayed than under immediate utilization. Dimensional comparison was an unlimited-capacity process under immediate utilization, but showed capacity limitations under delayed utilization.
\end{abstract}

Models of memory are concerned with the form in which incoming information is represented for internal maintenance (e.g., Anderson \& Bower, 1973; Kintsch, 1974; Melton \& Martin, 1972; Norman \& Rumelhart, 1975). Models of performance, on the other hand, are concerned with the way in which incoming information is utilized in carrying out particular tasks (e.g., Clark \& Chase, 1972; Kahneman, 1973; Keele, 1973; Posner, 1973; Carpenter \& Just, Note 1). The present study lies at the intersection of these two approaches to information processing. We employed speeded verification to examine some attributes of the internal representations of verbal descriptions and the manner in which the representations are compared to information extracted from pictures. A major goal was to investigate possible changes in encoding and comparison processes related to memory requirements placed on subjects during task performance.

We chose a verification task in which auditorily presented conjunctions which varied in polarity and syntax were compared to geometric figures differing in color and shape. Subjects performed the task under two conditions of storage demand or memory load.

This research, based on the first author's unpublished doctoral dissertation, was supported by NICHD Program-Project Grant 5P01-HD00973 to George Peabody College. Preparation of the manuscript was supported in part by NIH Postdoctoral Fellowship 1-F32-HD05157-01 from NICHD to the first author, under the sponsorship of Michael I. Posner. We would like to thank Drs. Susan W. Gray, Richard D. Sperber, Irving Biederman, and J. Kathryn Bock for their valuable comments and criticisms. Address correspondence to Thomas H. Carr, Department of Psychology, University of Oregon, Eugene, Oregon 97403.
In the first, a picture appeared immediately after the description was heard. This immediate utilization required the description to be briefly maintained with minimal interference from irrelevant factors. In the other storage condition, the picture did not appear for $4-5 \mathrm{sec}$ after the description ended. During that time, subjects performed a secondary counting task. The secondary task was introduced to place demands on the verbal rehearsal system which might force subjects to use an alternative or more efficient form of internal maintenance. Delayed utilization required a longer lasting representation which was immune to interference from irrelevant verbal activity, yet easily retrieved when the picture appeared.

Decision latencies in both conditions were measured from picture onset. We reasoned that main effects of delay on decision latency would constitute an overall measure of time needed to return processing resources from the secondary to the primary task. Increases in error rate with delayed utilization would reflect forgetting from temporary storage. Changes in representation to accommodate storage and retrieval demands would be identifiable as interactions involving delay with syntactic characteristics of the description. For example, if more complex constructions require more complex storage and retrieval operations, perhaps involving a more canonical storage format, one might expect increases in latency with delay for negatives relative to positives or for clauses relative to phrases. Resultant changes in comparison operations would appear as interactions involving delay with characteristics of the relation between description and picture. This relation was embodied in the correspondence between color and shape values specified by the description and values specified by the picture. 
License to make these interpretations follows from the choice of task. Most theorists require verbal and visual inputs to be parsed into similar forms before comparison across input modalities can be carried out (Anderson \& Bower, 1973; Clark \& Chase, 1972). This allows us to assume that encoding and comparison are in some sense separate components or stages of verification in this task. The use of conjunctive descriptions enables us to differentiate between characteristics of the description and characteristics of the description-picture relation. First, conjunctions allow the surface structure of the sentence to be varied without changing the dimensional information being specified. For example, "This is a green square" and "This is a square that's green" refer to the same geometric figure, but differ in syntactic organization. The structure of the description can therefore be manipulated independently of the relation between stimulus information referenced by the description and information presented in the picture. Second, conjunctions allow the correspondence between dimensional information specified by any given description and dimensional information specified by the picture to be varied along the two stimulus dimensions factorially. For example, "This is a blue cross" could be paired with a blue cross, a blue square, a green cross, or a green square. Description and picture would then match on both dimensions, match on color but mismatch on shape, match on shape but mismatch on color, or mismatch on both dimensions, respectively. In the absence of interactions between syntactic organization and dimensional correspondence, then, the above interpretations of interactions involving delay are straightforward. At the very least, any interactions involving dimensional correspondence can be attributed to comparison operations, since it would be difficult to argue that a property of the relation between stimuli, knowable only after comparing them, could affect a processing stage which must be completed prior to accomplishing the comparison.

Thus, two issues of representation will be addressed in this paper. The first is the extent to which internal representations are changed when they must be stored and retrieved for delayed utilization. These changes would occur as components of encoding, and would be inferred from interactions between syntactic organization or dimensional correspondence and delay. The second is the extent to which differences among descriptions defined by surface structure but irrelevant to objective reference are preserved in the internal representation and, therefore, effective in the comparison stage of verification. Effects related to surface-structure preservation would be inferred from interactions between syntactic organization and dimensional correspondence. For example, if order of presentation of dimensional information were preserved, one might expect that responses to mismatches on color alone would be faster for one syntactic organization, while responses to mismatches on shape alone would be faster for the other organization (see, e.g., Bacharach $\&$ Maisto, 1974). The nature of the interaction would indicate whether order affected comparison via primacy or recency.

Because decision latencies were examined as a function of dimensional correspondence, we will be able to draw some further conclusions about the dynamics of comparison operations, addressing the questions of serial vs parallel interrogation of dimensions and capacity demands made by comparison under light and heavy memory load. This will involve contrasts between processing time when only one stimulus dimension is discriminative with respect to the verification decision and processing time when both dimensions are discriminative.

\section{METHOD}

\section{Subjects}

Subjects were 20 paid volunteers from the academic community of George Peabody College and Vanderbilt University. All had normal or corrected-to-normal vision and hearing, and all were native speakers of English.

\section{Materials}

Verbal target descriptions of the forms "This (is, is not) a (blue, green) (square, cross)" and "This (is, is not) a (square, cross) that's (blue, green)" were recorded on one track of a tape using a Sony two-track stereo tape recorder. Sentences were spoken in a normal tone of voice. Care was taken not to place differential stress on color and shape that could lead subjects to infer topic-comment distinctions. A $10,000-\mathrm{Hz}$ pure signal was recorded on the other track of the tape. A brief interruption of the signal at the end of the last word of each target description was used to trigger solid-state and electromechanical apparatus used to present the visual stimuli and measure response latencies. Visual stimuli consisted of Kodachrome II color slides presented by a Kodak Carousel slide projector equipped with a tachistoscopic shutter. Each slide contained one geometric figure which could vary in color (blue, green) and shape (square, cross). Figures were cut from blue (No. 47, peak wavelength $=425$ nan) and green (No. 58, peak wavelength $=525$ nan) Kodak wratten gel filters, set into dark backgrounds, and backlit for photographing. The figures were approximately equal in total area and outside envelope dimensions, subtending about $3 \mathrm{deg}$ of visual angle horizontally and vertically when presented to the subject.

\section{Procedure}

Subjects heard the target descriptions through earphones. After each description, a slide containing one of the four figures appeared. Half the subjects received the slide immediately upon completion of the description, while the others received the slide after a delay of 4-5 sec. The length of the delay was varied randomly within this range to prevent subjects from establishing a set to begin response preparation before slide onset. The delay interval was filled by a secondary task which required subjects to count backward by threes from a random, two-digit number recorded on the tape $.5 \mathrm{sec}$ after the end of the description. The counting task was intended to load verbal short-term memory without creating task-specific interference.

All subjects received all possible combinations of description and figure. Each positive description was true of one figure and false of the others, while its corresponding negative was false of that figure and true of the others. Frequencies of occurrence of description-figure combinations were therefore adjusted 
to equate the probabilities of true and false responses to positive and negative descriptions over trials. Each subject responded six times to each of the 16 descriptions, for a total of 96 trials per subject. Thirty-two practice trials were given at the beginning of the subject's session, and a short break was given after 48 experimental trials. Two random orders were used, with half the subjects in each group receiving one order and half the other.

Subjects were instructed to indicate whether the description was true or false of the figure by pushing a response lever to the left or the right. Response directions were balanced across subjects and subjects operated the lever with the hand of their choice. Both speed and accuracy were emphasized in the instructions. Latencies were measured from slide onset with a Hunter 120-C Klockounter and were recorded by the experimenter. Each subject was informally interviewed after his or her session to solicit information about perceived task difficulty and strategic behavior.

\section{RESULTS}

\section{Primary Analysis}

A 2 by 2 by 2 by 2 analysis of variance was performed on median correct response latencies. Delay (immediate, delayed) was varied between groups, while polarity (positive, negative), response requirement (true, false), and syntactic organization (phrase, clause) were varied within subjects. In this analysis, response requirement was a measure of dimensional correspondence. Twelve observations per subject were possible in each treatment condition. At least nine correct responses in each condition were required for including data in the analysis. Error rates increased with response latencies across conditions. Rank-order correlations between error rates and latencies were calculated for the two between-groups conditions separately. The correlations were $+.86(\mathrm{df}=6)$ for immediate utilization and $+.66(\mathrm{df}=6)$ for delayed utilization. Table 1 shows error rates and latencies for all conditions in the primary analysis. It can be seen from the table that the probability of an error increased from an average of

Table 1

Response Latencies (in milliseconds) and Error Rates for All Conditions in the Primary Analysis

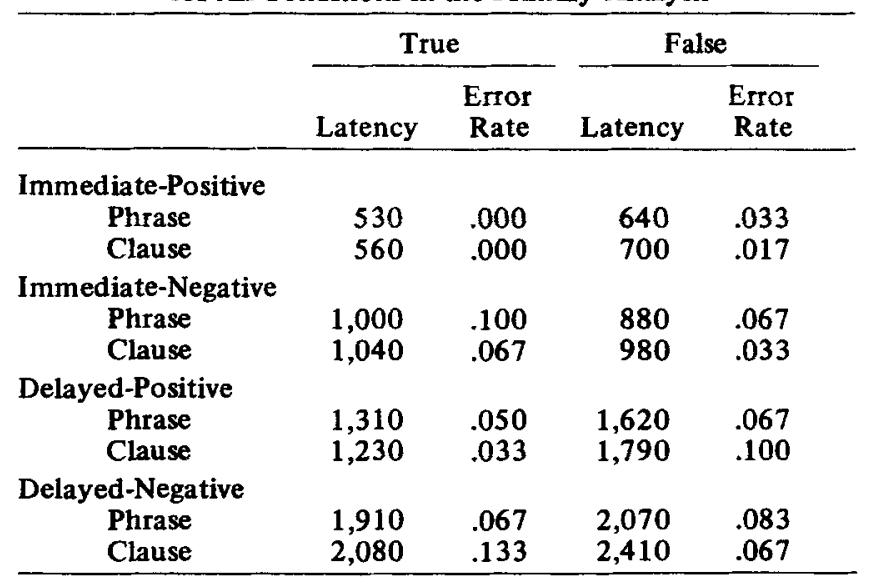

.039 in the immediate condition to an average of .073 in the delayed condition.

In the analysis of variance, main effects were found for all factors. Responses were faster in the immediate condition $(M=790 \mathrm{msec})$ than in the delayed condition $(M=1,800 \mathrm{msec})[F(1,18)=25.67$, $\mathrm{p}<.001, \quad \mathrm{MSe}=160,0001$. Responses to positive descriptions $(M=1,050 \mathrm{msec})$ were faster than responses to negative descriptions $(M=1,550 \mathrm{msec})$ $[\mathrm{F}(1,18)=35.41, \mathrm{p}<.001, \mathrm{MSe}=71,000]$. True responses $(M=1,210 \mathrm{msec})$ were faster than false responses $(M=1,390 \mathrm{msec})[F(1,18)=13.53, p<.005$, MSe $=9,000]$. Responses to phrase constructions ( $M=1,250 \mathrm{msec}$ ) were faster than responses to clause constructions $(\mathrm{M}=1,350 \mathrm{msec}) \quad[\mathrm{F}(1,18)=6.22$, $\mathrm{p}<.05, \mathrm{MSe}=7,000]$.

An interaction occurred between polarity and response requirement $[\mathrm{F}(1,18)=6.25, \mathrm{p}<.05, \mathrm{MSe}=6,000]$. Simple effects analyses showed that, for positive descriptions, true responses $(M=910 \mathrm{msec})$ were faster than false responses $(M=1,190 \mathrm{msec})$. For negative descriptions, there was no significant difference in latency between true responses $(M=1,510 \mathrm{msec})$ and false responses $(M=1,590 \mathrm{msec})$.

An interaction occurred between delay and response requirement $[F(1,18)=11.59, p<.005, M S e=9,000]$. Simple effects analyses showed that true responses were faster than false responses in both the immediate condition $\left(M_{F}=970 \mathrm{msec}, M_{T}=610 \mathrm{msec}\right)$ and the delayed condition $\left(M_{F}=2,120 \mathrm{msec}, \mathbf{M}_{T}=1,490 \mathrm{msec}\right)$. The increase in latency associated with false responses, however, was larger in the delayed condition $(D=630 \mathrm{msec})$ than in the immediate condition $(\mathrm{D}=360 \mathrm{msec}$ ).

Finally, a three-factor interaction occurred among delay, polarity, and syntactic organization $[F(1,18)=4.45, p<.05$, MSe $=2,000]$. Simple effects analyses were performed at each level of delay. In the immediate condition, responses were faster to positive descriptions $(M=610 \mathrm{msec})$ than to negative descriptions $(M=970 \mathrm{msec})$, and responses were faster to phrases $(M=760 \mathrm{msec})$ than to clauses $(M=820 \mathrm{msec})$. The same main effects occurred in the delayed condition, with faster responses to positive descriptions $(M=1,490$ $\mathrm{msec})$ than to negative descriptions $(M=2,120 \mathrm{msec})$ and faster responses to phrases $(M=1,730 \mathrm{msec})$ than to clauses $(M=1,880 \mathrm{msec})$. Polarity and organization interacted in the delayed condition, however, with a larger difference as a function of organization for responses to negative descriptions $(D=255 \mathrm{msec})$ than to positive descriptions $(D=40 \mathrm{msec}$, n.s.). Thus, syntactic organization affected latency for all but positive descriptions in the delayed condition.

\section{Secondary Analysis}

The primary analysis does not take into account the fact that positives could be false and negatives could 
be true in three ways: The dimensional information specified by the description could differ from the dimensional information specified by the picture in color only, in shape only, or in both. A 2 by 2 by 2 by 3 analysis of variance was performed on median correct response latencies from treatment conditions in which mismatches occurred between descriptive and pictorial dimensional information. Delay (immediate, delayed) was varied between groups. A hybrid response-requirement/polarity "factor" (false positive, true negative), organization (phrase, clause), and mismatch dimension (color, shape, both) were varied within subjects. Four observations per subject were possible in each treatment condition, and two correct responses per condition were required for data inclusion. Rank-order correlations between error rates and response latencies were $+.66(\mathrm{df}=10)$ for immediate utilization and $+.50(\mathrm{df}=10)$ for delayed utilization. Table 2 shows error rates and latencies for the conditions of the secondary analysis.

Main effects, mirroring those of the primary analysis, were found for the first three factors. Responses also differed as a function of mismatch dimension $[F(2,36)=4.19, \quad p<.05, \quad M S e=9,200]$, with responses to mismatches on both dimensions $(M=1,290 \mathrm{msec})$ faster than responses to color alone $(M=1,420 \mathrm{msec})$ or shape alone $(M=1,420 \mathrm{msec})$.

A marginally significant interaction occurred between delay and mismatch dimension $[F(2,36)=3.04, p<.06$, MSe $=9,200]$. Simple effects analyses showed no significant differences as a function of mismatch dimension for immediate utilization. For delayed utilization, responses to mismatches on both dimensions $(M=1,750 \mathrm{msec})$ were faster than responses to mismatches on either color alone $(M=2,010 \mathrm{msec})$

Table 2

Response Latencies (in milliseconds) and Error Rates for All Conditions in the Secondary Analysis

\begin{tabular}{|c|c|c|c|c|c|c|}
\hline & \multicolumn{6}{|c|}{ Mismatch Dimension } \\
\hline & \multicolumn{2}{|c|}{ Color } & \multicolumn{2}{|c|}{ Shape } & \multicolumn{2}{|c|}{ Both } \\
\hline & Latency & $\begin{array}{c}\text { Error } \\
\text { Rate } \\
\end{array}$ & Latency & $\begin{array}{c}\text { Error } \\
\text { Rate }\end{array}$ & Latency & $\begin{array}{r}\text { Error } \\
\text { Rate } \\
\end{array}$ \\
\hline & \multicolumn{6}{|c|}{ Immediate } \\
\hline $\begin{array}{c}\text { False-Positive } \\
\text { Phrase } \\
\text { Clause }\end{array}$ & $\begin{array}{l}660 \\
720\end{array}$ & $\begin{array}{l}.025 \\
.025\end{array}$ & $\begin{array}{l}680 \\
760\end{array}$ & $\begin{array}{l}.050 \\
.000\end{array}$ & $\begin{array}{l}620 \\
670\end{array}$ & $\begin{array}{l}.025 \\
.025\end{array}$ \\
\hline $\begin{array}{c}\text { True-Negative } \\
\text { Phrase } \\
\text { Clause }\end{array}$ & $\begin{array}{r}1,020 \\
950\end{array}$ & $\begin{array}{l}.150 \\
.025\end{array}$ & $\begin{array}{l}1,110 \\
1,110\end{array}$ & $\begin{array}{l}.100 \\
.125\end{array}$ & $\begin{array}{r}950 \\
1,050\end{array}$ & $\begin{array}{l}.050 \\
.050\end{array}$ \\
\hline & \multicolumn{6}{|c|}{ Delayed } \\
\hline $\begin{array}{c}\text { False-Positive } \\
\text { Phrase } \\
\text { Clause }\end{array}$ & $\begin{array}{l}1,760 \\
2,080\end{array}$ & $\begin{array}{l}.125 \\
.100\end{array}$ & $\begin{array}{l}1,660 \\
1,680\end{array}$ & $\begin{array}{l}.050 \\
.175\end{array}$ & $\begin{array}{l}1,480 \\
1,580\end{array}$ & $\begin{array}{l}.025 \\
.025\end{array}$ \\
\hline $\begin{array}{c}\text { True-Negative } \\
\text { Phrase } \\
\text { Clause }\end{array}$ & $\begin{array}{l}2,120 \\
2,070\end{array}$ & $\begin{array}{l}.100 \\
.175\end{array}$ & $\begin{array}{l}2,060 \\
2,350\end{array}$ & $\begin{array}{l}.050 \\
.175\end{array}$ & $\begin{array}{l}1,840 \\
2,110\end{array}$ & $\begin{array}{l}.050 \\
.150\end{array}$ \\
\hline
\end{tabular}

or shape alone $(M=1,940 \mathrm{msec})$, which did not differ significantly. Thus, a redundancy advantage obtained for delayed utilization.

\section{DISCUSSION}

While the pattern of results is complicated, two aspects of the data can be singled out immediately. First, the statistical independence of syntactic organization from both response requirement and mismatch dimension indicates that surface-structure variations among referentially equivalent descriptions did not play a role in the comparison stage of verification. We therefore assume that encoding operations eliminated referentially irrelevant structural features in representing the descriptions. Second, the interaction involving organization and polarity with delay indicated that encoding operations differed as a function of immediate vs delayed utilization. The interaction involving mismatch dimension and delay indicates that comparison operations also differed with delayed utilization. We will argue below that, while there is no evidence to suppose that syntax affected comparison, structural characteristics of the descriptions did play a large part in determining the time course and, therefore, the logistics of encoding. Encoding logistics became critical to successful performance in the dual-task environment of delayed utilization. Further, we will argue that the interactions involving polarity, a manipulation which affected both the syntactic complexity and the referential meaning of the descriptions, imply some rather sophisticated strategic adaptations to the processing requirements of the two utilization conditions. Finally, we will relate the effects of delay on comparison operations to increased capacity requirements of task performance in the delayed condition.

\section{Surface-Structure Preservation}

No interactions were found between syntactic organization and dimensional correspondence, either in the primary analysis, where correspondence was represented by response requirement, or in the secondary analysis, where correspondence was represented by mismatch dimension. We can conclude that differences in the syntactic organization of descriptions which did not change the dimensional information being specified had no effect on comparison. This conclusion extends to characteristics of organization such as the order in which dimensions are presented and possible differences in presuppositional status of dimensional information (Katz, 1973; Keenan, 1971). Either of these might be expected to produce directed attention effects during comparison were they preserved by encoding. We do not mean to claim that, after encoding, subjects had no knowledge of the surface structure they had processed. Knowledge of surface-structure organization, however, was not utilized in comparison operations. 
The absence of surface-structure effects on comparison implies that encoding operations parsed the two alternative syntactic organizations into similn $r$ representational formats for output to comparison operations. Because of differences in complexity which should have affected encodability, we therefore expected the clause construction to produce longer verification latencies than the phrase construction, at least when immediate utilization was required. This expectation was borne out in the main effect for organization found in both the primary and secondary analyses. Since organization as well as polarity interacted with delay, however, it is apparent that encoding operations, though not their completed representations, differed under immediate vs delayed utilization. It might be noted that this result is consistent with the more general assumption that internal representations are standardized or normalized across differences resulting from paraphrase (Kintsch, 1974; Norman \& Rumelhart, 1975). The finding contrasts at least superficially with predictions that might be inferred from Anderson and Bower (1973), since Human Associative Memory specifies different internal representations for phrase and clause constructions.

\section{Retrieval Time, Storage Form, and Forgetting}

Overall, as calculated from Table 1, decision latency increased an average of $1,010 \mathrm{msec}$ for delayed relative to immediate utilization. Since picture onset was not entirely predictable in the delayed condition, the increase should include most if not all the time necessary to retrieve the stored representation of the description, but may also include time to switch attention back to the primary task from the secondary task (see, e.g., Carr \& Bacharach, 1976; LaBerge, 1973; Moray, 1969). Therefore, system preparation plus retrieval appear to occupy a rather large part of total response time in the delayed condition. This may be related to the need for very accurate episodic computation during retrieval to avoid confusion with any lingering traces of previous descriptions not completely eliminated from temporary storage.

The interaction among polarity, organization, and delay shows that the increase in latency with delayed utilization varied as a function of the syntactic complexity of the description being processed, though the relation was not monotonic. Delay increased latency $880 \mathrm{msec}$ for both phrase- and clause-based positive descriptions, $1,050 \mathrm{msec}$ for phrase-based negatives, and 1,240 msec for clause-based negatives. Further, the difference in absolute latency between delayed phrase- and clause-based positives was not significant. Response time for delayed decisions was, therefore, affected by syntactic organization only for negative descriptions.

Perhaps, then, descriptions were parsed as much as possible before the secondary task began. This was time enough to complete parsing for positive descriptions, but not for negatives, which placed a larger load on encoding resources (Clark \& Chase, 1972; Trabasso, Note 2). An unparsed or partially parsed representation had to be stored for negative descriptions, and parsing completed after retrieval. For positives, a completely parsed representation was stored and comparison begun immediately upon retrieval. Since completely parsed representations did not distinguish between the original surface-structure organizations, no effect of syntactic organization appeared for delayed positives.

Turning to the question of forgetting, examination of error data indicates that memory for information contained in the description was quite good in the face of the secondary task. Mean error rate over the withinsubjects conditions increased from $3.9 \%$ for immediate utilization to $7.3 \%$ for delayed utilization. If the difference of $3.4 \%$ is taken as a maximum estimate of forgetting, temporary storage seems to have been very successful.

Subjective reports are consistent with the above conclusions. Subjects in the delayed condition reported that they were almost always able to remember the description and proceed to the picture, though the task demanded extreme effort and concentration. Most felt that negative descriptions were a greater challenge to memory than were positives, sometimes requiring verbatim reconstruction before attention could be turned to the picture. No one reported verbatim reconstruction for positive descriptions. Thus, subjects were aware of starting over from the beginning in verifying some of the delayed negative descriptions, supporting the inferences about storage and retrieval drawn from latency data.

\section{Optional Input Transformations}

So far, we have concluded that syntactic features of descriptions irrelevant to objective reference were not preserved by encoding processes, and that delayed or high-memory-load utilization forced changes in the logistics but not the output of encoding. There are several remaining characteristics of the data which indicate something more specific about the infrastructure of the representations sent on to comparison operations. The primary analysis showed that true responses to positive descriptions were faster than false responses, a common finding (Carpenter \& Just, 1975; Clark \& Chase, 1972; Trabasso, Note 2). However, no significant difference occurred between true and false responses to negative descriptions. The more usual result is that false negatives are verified faster than true negatives when the negative particle is retained in the internal representation, while true negatives are verified faster than false negatives when a transformation occurs which deletes the negative particle (Trabasso, Note 2). Our data fell between these two empirical outcomes, so we speculated that some subjects used the negative as received and others performed 
Table 3

Response Latencies (in milliseconds) as a Function of Polarity and Response Requirement for Immediate and Delayed Utilization

\begin{tabular}{|c|c|c|c|c|}
\hline & \multicolumn{2}{|c|}{ Immediate } & \multicolumn{2}{|c|}{ Delayed } \\
\hline & True & False & True & False \\
\hline $\begin{array}{l}\text { Positive } \\
\text { Negative }\end{array}$ & $\begin{array}{r}550 \\
1,020\end{array}$ & $\begin{array}{l}670 \\
930\end{array}$ & $\begin{array}{l}1,270 \\
2,000\end{array}$ & $\begin{array}{l}1,710 \\
2,240\end{array}$ \\
\hline
\end{tabular}

some transformation. Transformations, however, require the opportunity to carry them out. We therefore reasoned that transformations should have occurred more often in the delayed condition, with its .5 -sec free interval between the sentence and the number which initiated the counting task, than in the immediate condition. On this basis, we examined latencies as a function of polarity and response requirement for immediate and delayed utilization separately. As can be seen in Table 3, clear evidence in support of the hypothesis emerged. Apparently, only noise prevented a three-factor interaction involving polarity and response requirement with delay. The significant two-factor interaction in which the difference between true and false latencies was larger in the delayed than in the immediate condition supports this interpretation, since occurrence of a transformation would tend to lengthen the average false latency while shortening the average true latency.

Finally, if a transformation were used by some subjects but not by others, some evidence of bimodality should be discernible in the data of individual subjects. Table 4 shows that, under immediate utilization, three subjects evidenced faster true than false responses to negatives, while seven showed faster false than true responses. Under delayed utilization, six subjects showed an advantage for true responses, while four made faster false responses.

Empirical evidence is therefore available for an optional input transformation which deleted negative particles from descriptions prior to comparison operations. Such transformations have previously been reported only when stimuli varied along a single bipolar dimension, enabling unambiguous identification of an alternative target (Trabasso, Note 2). Since the stimuli used in the present experiment varied along two bipolar dimensions, negation could not entail an unambiguous alternative. It would be possible, however, to transform the negative conjunction into a positive disjunction. For example, a subject hearing "This is not a green square" could proceed as if verifying a description analogous to "This is either blue or a cross," responding "true" as soon as one match was found between a dimension specified by the disjunction and a dimension specified by the picture. Because a false response could not be executed until both description dimensions had been falsified by the picture, the disjunctive transformation would predict faster true than false responses to negative descriptions. In this way, correct responding after a disjunctive transformation requires a change only in decision criterion, not a change in comparison operations themselves. Thus employing the transformation would necessitate no modification of comparison dynamics.

It is not absolutely necessary that subjects be consciously aware of the occurrence of such a transformation process, but the case for the transformation would be strengthened if they were. One subject whose responses to true negatives were faster than his responses to false negatives did spontaneously report using a disjunctive strategy. To date, the occurrence of input transformations has been attributed primarily to favorable information conditions: When stimuli are characterized by bipolar opposition along a single dimension, transformations appear (Trabasso, Note 2). Our data indicate that the aptitudes and motivations of individual subjects relative to task characteristics may be more important than stimulus characteristics perse in determining the occurrence of optional input transformations. The motivation behind this particular transformation is somewhat obscure, however. Looking again at Table 4, transforming negative conjunctions into positive disjunctions accrued no consistent benefit either to verification time or accuracy. Averaged across all conditions, the mean latency to negatives for subjects showing the transformation was $1,390 \mathrm{msec}$, while the mean latency for subjects not showing the transformation was $1,420 \mathrm{msec}$. The difference of $30 \mathrm{msec}$ is not very large, given the variability of our data. Error rates are also nearly identical, subjects showing the transformation averaging $6.6 \%$ errors on negatives and subjects not showing the transformation averaging $6.9 \%$ errors. The only reason left for undertaking the transformation appears to be relief from the subjective burden of processing negative information.

Table 4

Response Latencies (in milliseconds) for Subjects Showing Evidence For and Against the Disjunctive Transformation

\begin{tabular}{|c|c|c|c|c|c|c|}
\hline & \multicolumn{3}{|c|}{ Immediate Negatives } & \multicolumn{3}{|c|}{ Delayed Negatives } \\
\hline & $\mathbf{n}$ & True Response & False Response & $\mathbf{n}$ & True Response & False Response \\
\hline $\begin{array}{l}\text { True }<\text { False (transformed) } \\
\text { False }<\text { True (untransformed) }\end{array}$ & $\begin{array}{l}3 \\
7\end{array}$ & $\begin{array}{r}710(.028) \\
1,140(.107)\end{array}$ & $\begin{array}{l}870(.014) \\
950(.065)\end{array}$ & $\begin{array}{l}6 \\
4\end{array}$ & $\begin{array}{l}1,780(.125) \\
1,850(.052)\end{array}$ & $\begin{array}{l}2,200(.099) \\
1,740(.052)\end{array}$ \\
\hline
\end{tabular}

Note-The figures in parentheses are error rates 


\section{Comparison Dynamics and Processing Capacity}

While we cannot discriminate unambiguously between serial and parallel interrogation of dimensional information during comparison, we can draw some firm conclusions about subjects' abilities to meet capacity demands made by comparison operations, and offer some speculations about serial vs parallel processing. The fact that latency did not vary with mismatch dimension under immediate utilization indicates that speeded verification carried out as a single task did not exceed the processing capacity which subjects were willing and able to allocate to the task. The appearance of a redundancy gain under delayed utilization, however, indicates that adding the secondary task caused capacity requirements to outstrip available resources, even though concurrent performance was not required. The resulting limited-capacity processing characteristics did not extend to preferential interrogation of one dimension over the other. The empirical absence of dimensional preference in either storage condition, and the logical simplicity of parallel relative to serial unlimited-capacity models (Townsend, 1974), cause us to choose parallel interrogation of dimensional information during comparison operations, at least for immediate utilization. In delayed utilization, parallel processing could also have occurred, but with unequal allocation of processing resources between the two dimensions. On each trial, a dimension chosen at random was favored with the normal resource allotment. The second dimension received what was left over, and was processed at a slower rate until the favored dimension was finished. We realize, however, that a switch to serial interrogation with random access to dimensions would have produced the same pattern of results. Either seems a reasonable adaptation to capacity limitations imposed by the dual-task conditions of delayed utilization.

\section{CONCLUSIONS}

In the present study, we have seen that people can verify two-term conjunctive descriptions against two-dimensional geometric figures with minimal loss of accuracy under conditions of high memory load, though overcoming memory load taxes available processing capacity and increases total processing time. People appear to use rather similar internal representations of descriptions in carrying out comparisons under low and high mernory load. Because encoding time varies with surface-structure organization but comparison time does not, it appears that the representations do not preserve surface-structure information beyond the minimum necessary for objective reference. These characteristics of the representation are congruent with theoretical assumptions made by Kintsch (1974) and Norman and Rumelhart (1975) about the relative importance of semantics and syntax as determinants of encoding operations.
Perhaps the most interesting finding of the present study, however, is the discovery of an optional input transformation, carried out by some subjects but not by others, whose sole advantage seems related to motivational rather than processing factors. Many investigators have reported the objective difficulty people have in understanding and remembering negative information. Objective difficulty is apparently accompanied by subjectively motivated strategies intended to make cognitive performance more palatable, whether or not the task becomes any easier.

\section{REFERENCE NOTES}

1. Carpenter, P. A., \& Just, M. A. Sentence comprehension: An information-processing model of verification. Complex Information Processing Working Paper 246. Pittsburgh: Carnegie-Mellon University, 1974.

2. Trabasso, T. Reasoning and the processing of negative information. Invited address to Division 3 of the Annual Meeting of the American Psychological Association, September 1970.

\section{REFERENCES}

Anderson, J. R., \& Bower, G. H. Human associative memory. Washington, D.C: Winston, 1973.

Bacharach, V. R., \& Maisto, A. A. Prenominal adjective order and visual discrimination in children. Journal of Experimental Child Psychology, 1974, 17, 495-506.

Carpenter, P. A., \& Just, M. A. Sentence comprehension: A psycholinguistic model of sentence verification. Psychological Review, 1975, 82, 45-73.

Carr, T. H., \& Bacharach, V. R. Perceptual tuning and conscious attention: Systems of input regulation in visual information processing. Cognition: International Journal of Cognitive Psychology, 1976, 4. 281-302.

Clark, H. H., \& Chase, W. G. On the process of comparing sentences against pictures. Cognitive Psychology, 1972, 3, 472-517.

Kahneman, D. Attention and effort. Englewood Cliffs, N.J: Prentice-Hall, 1973.

KATz, J. J. On defining "presupposition." Linguistic Inquiry, $1973,4,256-260$.

KeEle, S. W. Attention and human performance. Pacific Palisades, Calif: Goodyear, 1973.

KeEnaN, E. L. Two kinds of presupposition in natural language. In C. J. Fillmore \& D. T. Langendoen (Eds.), Studies in linguistic semantics. New York: Holt, 1971.

KINTSCH, W. The representation of meaning in memory. Hillsdale, N.J: Erlbaum, 1974.

LABERGE, D. Identification of two components of the time to switch attention. In S. Kornblum (Ed.), Attention and performance IV. New York: Academic Press, 1973.

Melton, A. W., \& Martin, E. Coding processes in human memory. Washington, D.C: Winston, 1972.

Moray, N. Attention: Selective processes in vision and hearing. London: Hutchinson, 1969.

Norman, D. A., \& Rumelfart, D. E. Explorations in cognition. San Francisco: Freeman, 1975.

Posner, M. I. Cognition: An introduction. Glenview, 1ll: Scott Foresman, 1973.

Townsend, J. T. Issues and models concerning the processing of a finite number of inputs. In B. Kantowitz (Ed.), Human information processing: Tutorials in performance and cognition. Hillsdale, N.J: Erlbaum, 1974.

(Received for publication August 30, 1976; revision accepted January $12,1977$. 